\title{
VLHL plasminogen activator inhibitor spontaneously reactivates from the latent to active form
}

\author{
JERZY JANKUN ${ }^{1,2}$, ANSARI M. ALEEM ${ }^{1}$, STEVE H. SELMAN ${ }^{1}$, \\ VENKATESHA BASRUR ${ }^{3}$ and EWA SKRZYPCZAK-JANKUN ${ }^{1}$ \\ ${ }^{1}$ Urology Research Center, Department of Urology, Health Science Campus, Toledo, OH, USA; \\ ${ }^{2}$ Department of Clinical Nutrition, Medical University of Gdansk, Gdansk, Poland; \\ ${ }^{3}$ Department of Pathology, University of Michigan, Ann Arbor, MI, USA
}

Received August 26, 2008; Accepted September 22, 2008

DOI: 10.3892/ijmm_00000101

\begin{abstract}
Wild-type plasminogen activator inhibitor type 1 (PAI-1) is a fast-acting uPA and tPA inhibitor with half-life of 1-2 h. Recombinant PAI-1 with two mutations, Q197C and G355C, shows a very long half-life (VLHL). An introduced disulfide bridge holds together two central, parallel strands of $B$-sheet $A$, preventing their separation to incorporate residues $\mathrm{P} 4-\mathrm{P} 14$ during the serpin's transition into latency. An active PAI-1 is usually described as a single structure with the reactive center loop (RCL) with P1-P1' (R369-M370) extended far from the bulk of the serpin's body. We have found that VLHL PAI-1 exists in several active forms that travel with different electrophoretic mobilities. Under aerobic conditions, two distinct active forms are observed. Upon reduction of cysteines, the VLHL mutant converts into the latent form, which spontaneously reactivates into a fully or partially active serpin, with yet another mobility. Utilizing electrophoresis, zymography (to check PAI-1 activity toward uPA) and theoretical calculations for molecular modeling, we have characterized active 1,2, 3 and latent conformers of VLHL PAI-1 and their behaviors at normal and elevated temperatures, and in normal or reducing environments. VLHL PAI-1 activity is not affected, and the molecules do not polymerize unless reduced and/or heated. VLHL PAI-1 associates into dimers and bigger oligomers when -SH groups become available for oxidation and formation of intra- or intermolecular-S-S- bridges between conformers of different shapes and activities. We postulate that the active structures differ in RCL conformation and their position in relation to the gate region and the rest of the molecule.
\end{abstract}

Correspondence to: Dr Jerzy Jankun, Urology Research Center, University of Toledo, Health Science Campus, Mail Stop 1091, 3065 Arlington, Toledo, OH 43614-5807, USA

E-mail: jerzy.jankun@utoledo.edu

Key words: very long half-life, plasminogen activator inhibitor type one, PAI-1 mutation

\section{Introduction}

Plasminogen activator inhibitor type one (PAI-1) is a specific and fast-acting inhibitor of tissue type (tPA) and urokinase type (uPA) plasminogen activators. Under normal physiological conditions PAI-1 provides the equilibrium between blood clot formation and blood clot lysis by reacting with tPA. Also, by regulating uPA activity, it controls wound healing and angiogenesis $(1,2)$. The uPA- or tPA-driven proteolytic activity is upregulated in some diseases as can be seen in cases of atherosclerosis, abnormal bleeding, cancer or other ailments $(3,4)$. Thus, inhibition of $\mathrm{UPA}$ or tPA by PAI- 1 can be exploited as a possible therapeutic measure.

PAI-1 is synthesized as an active molecule that spontaneously converts into a latent form (with a half-life of 1-2 h) that cannot inhibit uPA or tPA. In the active form, the reactive center loop ( $\mathrm{RCL}$ ) that contains the $\mathrm{P} 1-\mathrm{P} 1$ ' site projects outward from the body of the molecule with $\mathrm{P} 1 \sim 55 \AA$ away from its position in the latent form (according to crystal structures, PDB entries 1b3k, 1dvm and 1c5g). Arg369 at this site binds to and inactivates TPA or UPA. In latent PAI-1, this loop is retracted and becomes inserted into $B$-sheet $\mathrm{A}$, making the P1-P1' site inaccessible to plasminogen activators (5-7).

This $45 \mathrm{kDa}$ protein with 402 amino acids belongs to the serpin superfamily and contains only a single cysteine (Cys9) $(8,9)$. However, most of the studied recombinant PAI-1 constructs lack the first 23 amino acids, including the only cysteine of wild-type PAI-1 $(5,6,8)$. The lack of disulfide bridges is one of the factors that allows such flexibility for the PAI-1 molecule during the active to latent form conversion. The highly bendable RCL accounts for rapid tPA and uPA inhibition but also supports prompt conversion into the stable latent form (10).

By restricting movement of the central strands in B-sheet A, as well as limiting the flexibility of the helix D region, it is possible to prevent/limit insertion of the reactive loop and hence extend the half-life of PAI-1 (6). Based on the known structure of active PAI-1, we have identified amino acids that can be substituted with cysteine residues to produce disulfide bridges that can constrain PAI-1 into a stable form that remains active for a prolonged period of time. We have previously identified seven possible cysteine mutants (2-6 point 
mutations, with an even number of cysteines) at the top and bottom portions of strands 341-352 and 185-197 and within the helix D region or a combination thereof as potential candidate sites. The longest lasting half-life was observed for a B-sheet top PAI-1 mutant (Cys197, Cys355), which was named VLHL PAI-1 (very long half-life). We have measured its activity for up to $700 \mathrm{~h}$, and by extrapolating these results, we have determined that the half-life for this mutant could reach over 9 months.

When purified, this protein can be seen on a gradient (412\%) SDS gel as two distinct bands (Fig. 1). One was defined as the active and the other as the latent form of VLHL PAI-1 $(2,11)$. Unexpectedly, we have found that as time passes, the band of active VLHL PAI- 1 becomes dominant and the latent form diminishes. This observation has prompted more detailed investigation of this phenomenon, which has revealed that indeed inactivated VLHL PAI-1 with reduced disulfide bridges could spontaneously convert into the active form in aerobic conditions.

\section{Materials and methods}

Obtaining the starting structure. PAI-1 structure visualization was done on a PC using the PyMol program $(12,13)$. Atomic coordinates of PAI-1 were retrieved from the Protein Data Bank, entries $1 \mathrm{~b} 3 \mathrm{k}, 1 \mathrm{dvm}$ and $1 \mathrm{c} 5 \mathrm{~g}$ depicting the structures of the active (14) and latent (15) forms. Coordinates of wtPAI-1 structures during the transition were based on molecular modeling done by Krüger et al (16). However, the atomic coordinates provided by the authors illustrate wide opening of $B$-sheet $A$ as the first step followed by rearrangement of the RCL that attaches on top of this sheet rather than being fully incorporated into it. In our modeling, we have assumed an orchestrated, simultaneous movement of the secondary structure elements involved, ending as the latent form documented in the crystal structure.

Macromolecular docking using spherical polar fourier correlations. Molecular modeling of docking VLHL PAI-1 conformers was done by Hex v. 4.5 essentially as described by Mustard and Ritchie (17) and Ritchie and Kemp (18) the program manual (http://www.csd.abdn.ac.uk/hex/. For docking, we have used active 1,2,3 and latent conformers of VLHL PAI-1.

Purification of VLHL PAI-1. We have employed a baculovirus expression system basically as described before (11). The supernatant from lysed cells was loaded onto a column packed with nickel resin (Invitrogen) at a flow rate of $0.3 \mathrm{ml} /$ min (GradiFrac system, Pharmacia Biotech). The column was then washed with wash buffer containing $40 \mathrm{mM}$ imidazole in native buffer at a flow rate of $1 \mathrm{ml} / \mathrm{min}$ until no protein was detected. The protein was then eluted from the column using a gradient of $40-250 \mathrm{mM}$ imidazole in native buffer at a flow rate of $1 \mathrm{ml} / \mathrm{min}$. The peak fractions were collected and concentrated. VLHL PAI-1 was further purified by HPLC. The affinity purified VLHL PAI-1 (eluted in $50 \mathrm{mM} \mathrm{NaH}_{2} \mathrm{PO}_{4}$ $\mathrm{pH} 8.0,0.5 \mathrm{M} \mathrm{NaCl}$ and $250 \mathrm{mM}$ imidazole) was subjected to size exclusion chromatography using a Superose 12 FPLC column (ID\#9748048, Pharmacia Biotech). The protein was eluted with the column equilibration buffer $\left(50 \mathrm{mM} \mathrm{NaH} \mathrm{PO}_{4}\right.$ $\mathrm{pH} 8.0$ and $0.5 \mathrm{M} \mathrm{NaCl}$ ) at a flow rate of $0.5 \mathrm{ml} / \mathrm{min}$ and was concentrated using a Vivaspin column (10000 MWCO). The purity of VLHL PAI- 1 was determined to be $+95 \%$ by PAGE densitometry measurements (11).

In-gel digestion with trypsin. The proteins stained with Coomassie blue were excised from the $4-12 \%$ gradient SDS PAGE gel and destained with $30 \%$ methanol for $3 \mathrm{~h}$ at room temperature. In-gel proteolysis with modified, sequencing grade trypsin (Promega, Madison, WI) was done essentially as previously described (19).

Peptide sequencing using liquid chromatography/tandem mass spectrometry (LC-MS). Two microliters of the digest were separated on a reverse phase column (Aquasil C18, $15 \mu \mathrm{m}$ tip x $75 \mu \mathrm{m}$ id x $5 \mathrm{~cm}$ Picofrit column, New Objectives, Woburn, MA) using an acetonitrile/1\% acetic acid gradient system (5-75\% acetonitrile over $35 \mathrm{~min}$ followed by $95 \%$ acetonitrile wash for $5 \mathrm{~min}$ ) at a flow rate of $250 \mathrm{nl} / \mathrm{min}$. Peptides were directly introduced into a linear ion-trap mass spectrometer (LCQ-Deca, ThermoFinnigan) equipped with a nano-spray source. The mass spectrometer was set for analyzing the positive ions and acquiring a full MS scan $(400-2000 \mathrm{~m} / \mathrm{z})$ and data dependent MS/MS spectra on the top 4 ions (collision energy $\sim 35 \%$ ). Dynamic exclusion was set to acquire two MS/MS scans for each ion and exclude it for a further $2 \mathrm{~min}$. Peptide sequences were identified by searching the MS/MS data against the human IPI protein database (v3.32) and reverse database using X!Tandem with the k-score plug-in (Open source search engine developed by Global Proteome Machine). The search was restricted to tryptic peptides with 1 missed cleavage and a mass tolerance window of $3 \mathrm{Da}$ for the parent and $0.8 \mathrm{Da}$ for the fragment ions. Results obtained were subjected to TransProteomic Pipeline (TPP), a suite of software that includes PeptideProphet and ProteinProphet. Only proteins that were retained with a ProteinProphet probability of $>0.9$ (error rate $<2 \%$ ) were considered as positive.

Reverse zymography. VLHL PAI-1 activity was determined by reverse zymography (two-gel system) essentially as described by Tozser and Berta (20) with some modifications as described below. Different conformers of VLHL PAI-1 were separated on a gradient (4-12\%), non-reducing SDS gel at $4^{\circ} \mathrm{C}$. After electrophoresis, the gel was incubated at room temperature twice for $45 \mathrm{~min}$ in renaturation buffer $(2.5 \%$ Triton X-100 in water to remove SDS) with gentle agitation. An indicator gel was prepared by mixing $12 \mathrm{ml}$ of $1.4 \%$ agarose in $0.01 \mathrm{M}$ phosphate-buffered saline (PBS) $\mathrm{pH} 7.4$ at $50^{\circ} \mathrm{C}$ with $5 \mathrm{ml}$ of $4 \%$ non-fat powdered milk in $0.01 \mathrm{M}$ PBS $\left(\mathrm{pH} 7.4,37^{\circ} \mathrm{C}\right)$, containing $7 \mu \mathrm{g} / \mathrm{ml}$ human plasminogen and $13 \mathrm{IU} / \mathrm{ml}$ human uPA (both from American Diagnostica Inc.). The gel was cast in a $7 \times 10 \mathrm{~cm}$ mold. After the indicator gel set, excess fluid from the top of the SDS gel was removed with a paper towel and then it was laid on the indicator gel. The unit was sealed in plastic wrap and incubated in a moist chamber at $37^{\circ} \mathrm{C}$ for $16 \mathrm{~h}$, after which non-lysed bands of milk protein overlaying the active VLHL PAI-1 were clearly visible. Both gels were stained with Coomassie stain. The 

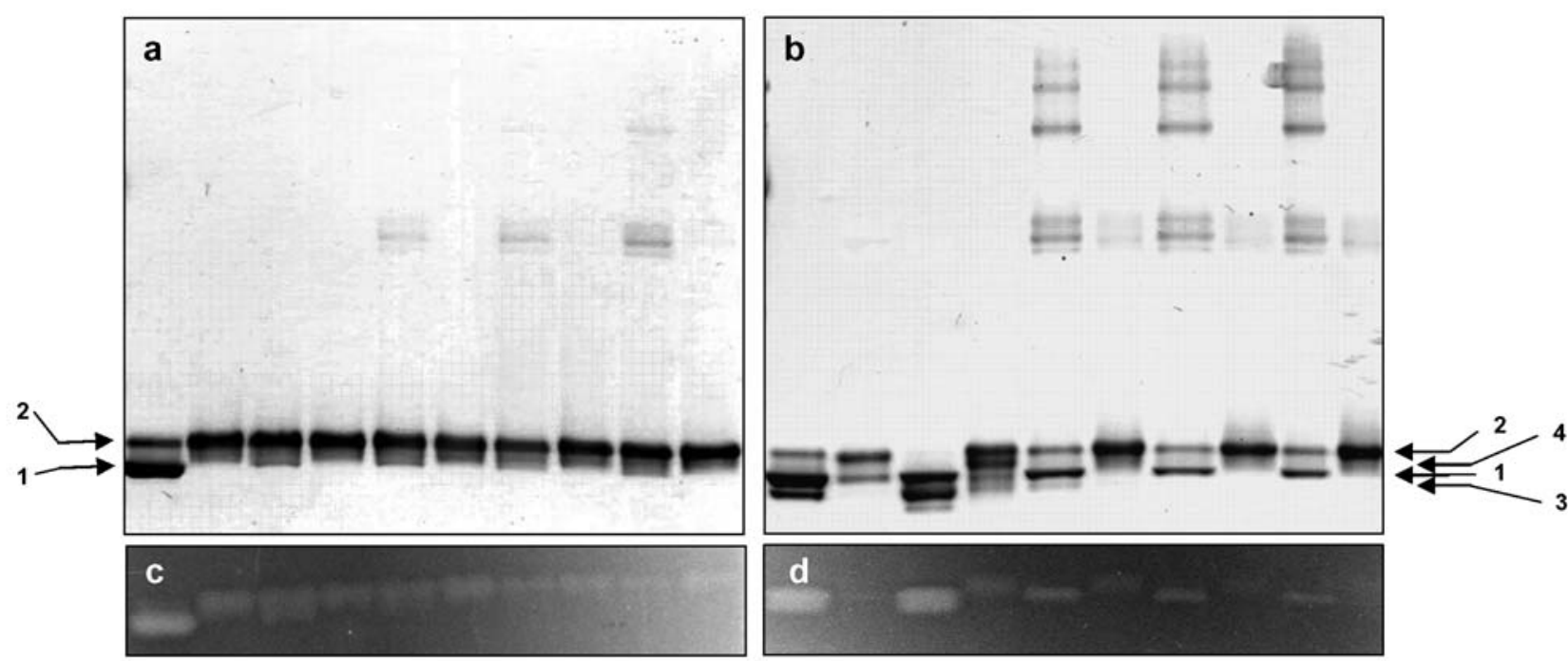

\begin{tabular}{|c|c|c|c|c|c|c|c|c|c|c|c|c|c|c|c|c|c|c|c|}
\hline lane & 1 & 2 & 3 & 4 & 5 & 6 & 7 & 8 & 9 & $\begin{array}{l}1 \\
0\end{array}$ & $\begin{array}{l}1 \\
1\end{array}$ & $\begin{array}{l}1 \\
2\end{array}$ & $\begin{array}{l}1 \\
3\end{array}$ & $\begin{array}{l}1 \\
4\end{array}$ & $\begin{array}{l}1 \\
5\end{array}$ & $\begin{array}{l}1 \\
6\end{array}$ & $\begin{array}{l}1 \\
7\end{array}$ & $\begin{array}{l}1 \\
8\end{array}$ & $\begin{array}{l}1 \\
9\end{array}$ \\
\hline Day & 0 & 0 & 0 & 0 & 0 & 0 & 0 & 0 & & 0 & 7 & 7 & 7 & 7 & 7 & 7 & 7 & 7 & 7 \\
\hline DTT & - & + & $+1-$ & + & * & $\begin{array}{l}+ \\
*\end{array}$ & $\bar{*}$ & $\begin{array}{l}+ \\
\text { * }\end{array}$ & * & $\begin{array}{l}+ \\
\text { * }\end{array}$ & - & + & $+1-$ & + & it & $\underset{* *}{+}$ & $\dot{\bar{*}}$ & $\underset{\star \star}{+}$ & $\overline{\star \star}$ \\
\hline $\begin{array}{l}\text { Tempe- } \\
\text { rature } \\
{ }^{\circ} \mathrm{C}\end{array}$ & $\begin{array}{l}2 \\
0\end{array}$ & $\begin{array}{l}2 \\
0\end{array}$ & $\begin{array}{l}2 \\
0\end{array}$ & $\begin{array}{l}2 \\
0\end{array}$ & $\begin{array}{l}6 \\
0\end{array}$ & $\begin{array}{l}6 \\
0\end{array}$ & $\begin{array}{l}8 \\
0\end{array}$ & $\begin{array}{l}8 \\
0\end{array}$ & $\begin{array}{l}0 \\
0\end{array}$ & $\begin{array}{l}1 \\
0 \\
0\end{array}$ & $\begin{array}{l}2 \\
0\end{array}$ & $\begin{array}{l}2 \\
0\end{array}$ & $\begin{array}{l}2 \\
0\end{array}$ & $\begin{array}{l}2 \\
0\end{array}$ & $\begin{array}{l}6 \\
0\end{array}$ & $\begin{array}{l}6 \\
0\end{array}$ & $\begin{array}{l}8 \\
0\end{array}$ & $\begin{array}{l}8 \\
0\end{array}$ & $\begin{array}{l}1 \\
0 \\
0\end{array}$ \\
\hline
\end{tabular}

Figure 1. SDS PAGE gel of (a) freshly purified VLHL PAI-1 and (b) VLHL PAI-1 stored for 7 days and reverse zymography of (c) freshly purified VLHL PAI-1 and (d) VLHL PAI-1 stored for 7 days. *Indicates sample 3, from which DTT was removed, heat treated and some protein polymerized, while subsequent treatment with DTT depolymerized them; ${ }^{* *}$ indicates the same as * but samples were stored for 7 days and treated with DTT at day 7 . Arrows point to 1) active 1 VLHL PAI-1;2) latent VLHL PAI-1; 3) active 2 VLHL PA-1 and 4) active 3 VLHL PAI-1.
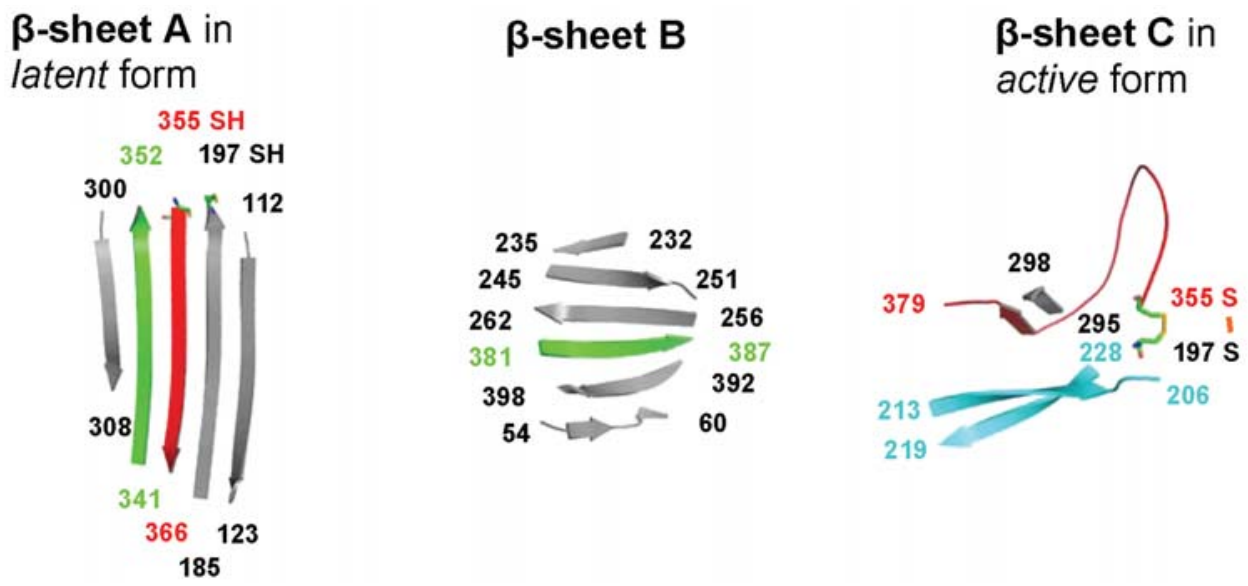

Figure 2. The structure of $\beta$-sheets $\mathrm{A}, \mathrm{B}$ and $\mathrm{C}$ and their sequential numbering.

SDS gel was stained for $1 \mathrm{~h}$, whereas the indicating gel was stained for $4 \mathrm{~h}$. Both the gels were destained with water and photographed. During incubation, proteins of milk from the indicating gel diffuse into the SDS gel and were detected with Coomassie blue as a uniform bluish stain through the gel. Therefore, a parallel gel was run at the same time to detect fine bands of multiple conformers of VLHL PAI-1 and these are shown in Figs. 1 and 3.

\section{Results and Discussion}

Freshly purified VLHL PAI-1 can be detected on an SDS PAGE gel as two distinct bands (Fig. 1, lane 1): 'band 1' corresponds to the active form and 'band 2' to the latent form of VLHL PAI-1. The optical densities of the protein bands were estimated as $70 \%$ active (band 1) and $30 \%$ of latent (band 2), but this ratio can change from one purification to another. VLHL PAI-1 inhibits UPA as determined by the amidolytic assay and forms a complex with uPA (data not shown), which is consistent with our previous reports $(2,6,11)$. Treatment of VLHL PAI-1 with dithiothreitol (DTT) reduces disulfide bridges, and SDS PAGE gel (Fig. 1, lane 2) shows a shift of the dominant band to the position previously described as the latent form of VLHL PAI-1 $(2,11)$. This shift was observed shortly after DTT treatment ( 30 min), which is too rapid for a full conversion of VLHL PAI-1 from 
the active to latent form, since PAI-1 has a half-life of $\sim 2 \mathrm{~h}$. This raises the possibility that VLHL PAI-1, in addition to the active and latent forms, has some intermediate conformers that migrate on the SDS PAGE gel with the same or similar electrophoretic mobility as active and latent VLHL PAI-1.

To establish which bands of VLHL PAI-1 on the gel retain the anti-uPA activity, we have performed a reverse zymography. As seen in Fig. 1b, band 1 prevents lysis of casein by uPA activated plasmin. This band has been described as the active PAI- 1 by us and others $(2,11,21)$. Unexpectedly, band 2 showed anti-uPA activity as well (Fig. 1a, lane 2, band 2). This band is described in the literature as non-active PAI-1 $(21,22)$. Subsequent removal of DTT has not significantly changed the appearance of bands on SDS gel (Fig. 1a, lane 3 ), with the exception of a lower band becoming somewhat more visible, but it has shown anti-uPA activity in reverse zymography. An additional treatment of this sample with DTT reversed this effect (Fig. 1a, lane 4).

VLHL PAI-1 stability over 7 days. The appearance of VLHL PAI-1 stored at room temperature on SDS gel [Fig. 1, lane 11 (first on panel c), bands 1, 2,3] is different than its counterpart analyzed shortly after purification (panel a, lane 1). Three bands can be detected, with bands 1 and 3 determined to be 'active' by reverse zymography. Also, these active bands are dominant, constituting $\sim 90 \%$ as estimated by densitometry of the SDS gel. A sample treated at day 0 with DTT and stored for 7 days lost its activity in the zymography experiment (Fig. 1, lane 12). The same sample from which DTT was removed at day 0 converted into the active form during 7 days of incubation at room temperature and showed three 'active' bands (Fig. 1, lane 13). The active sample of VLHL PAI-1 from lane 13 that was treated again with DTT showed only trace activity (Fig. 1, lane 14). All of these data indicate that VLHL PAI-1 exists as multiple conformers of which some are active and some are not.

Protein identification by peptide sequencing using liquid chromatography-tandem mass spectrometry. A total of four bands were excised from the gel. As shown in Fig. 1, bands marked 1-4 were analyzed. All of them were identified as PAI-1, and no other contaminating proteins were detected.

Latency transition of VLHL PAI-1. Multiple bands on an SDS gel clearly indicate that there are different conformers coexisting in the solution. Transition from the active to latent form of wild-type PAI-1 implies significant rearrangement of the protein molecule. We have analyzed this process based on the molecular modeling of transitions and the atomic coordinates provided by Dr P. Krüger (16).

The predominant change in the PAI-1 molecule usually described in the literature is the sliding of a part of the reactive center loop into the middle of $B$-sheet A. Although expansion of this sheet might be the most noticeable difference, the PAI-1 molecule has three B-sheets [Fig. 2A, the longitudinal sheet; $\mathrm{B}$, the horizontal sheet in the back, perpendicular to $A$, and $C$, the sheet containing a long $B$-finger above the other two (A and $\mathrm{B}$ ) and below the RCL] and all three of them, as well as other fragments of the molecular structure, are affected by the transition between the active and latent forms. The RCL stretches from 355 to 381 with Arg369 and Met370 being P1-P1'. The preceding fragment of the $\mathrm{N}_{\mathrm{t}}-\mathrm{RCL}$ is built into sheet $\mathrm{A}$ in the parallel orientation on one side $(\downarrow \uparrow \uparrow)$, while the fragment following the $\mathrm{C}_{\mathrm{t}}-\mathrm{RCL}$ is firmly held in the middle of sheet B (Fig. 2, green arrows) in the anti-parallel orientation $(\downarrow \uparrow \downarrow)$. The strands of sheet A $(\uparrow \uparrow)$ have to move apart to insert 355-366, upon which the sheet becomes anti-parallel $(\uparrow \downarrow \uparrow)$. This movement of the strands in sheet $\mathrm{A}$ requires the rearrangement of the hydrophobic core between sheets $\mathrm{A}$ and $\mathrm{B}$ and some changes in hydrophilic interactions as well. For example, in the active molecule, Tyr252 is stabilized by the aromatic moieties of Trp198 and Phe194, but in the latent form its $\mathrm{OH}$ group makes a strong hydrogen bond interaction with OG of Thr356. Also, the side chains forming a salt bridge between His252 and Glu112 change conformation with the sheet A expansion, but still maintain the junction between $B$-sheets $A$ and $B$. The RCL has to break its interaction with sheet $\mathrm{C}$, swing over a flexible B-finger (blue on Fig. 2) and pass through a gate formed by its coiled tip (213-219) and a turn between strand in sheet B and a following helix (264-270). It could be a tautomeric change in Arg379-Pro380 located in the hinge that plays a crucial role in this transformation. It can bend the main chain in the opposite direction, bringing Arg379 near Asp216 and Asp52 to stabilize the $\mathrm{C}_{\mathrm{t}}$-hinge of the RCL during this transition. Meanwhile, its $\mathrm{N}_{\mathrm{t}}$-end (356-363) interacts in the anti-parallel orientation with 206-199 when the RCL glides over sheet $\mathrm{C}$ before collapsing into sheet $\mathrm{A}$. An important step has to be passing over the coil of 212-219 when the RCL residues Arg379 and Glu374 can be attracted by nearby hydrophilic residues, making contacts between Arg379...Asp52... Lys214 and Glu374...Arg53...Asp216 that can stabilize the intermediate structure(s) when the PAI-1 molecule can still exhibit activity (Fig. 2b, active 2). After collapsing and binding the chain of 355-366 inside sheet A, the active site Arg369 is on the outskirts of the molecule but close to its bulk and can be engaged in a salt bridge with Asp181, thus precluding insertion into a specificity pocket of a designated protease. In the latest publication on the structural differences between active forms of PAI-1 (23) the authors point out that in contrast to crystal structures describing stabilized 4-point mutant, the proximal hinge of RCL in wild PAI-1 is closer to the latent than active form and has partially open, a pigeon-toed conformation in B-sheet A with partially inserted loop like other native serpins. They also suggest that RCL could be closer to the molecular body in wPAI-1 than its extended conformation observed in the crystal of the stabilized 4-point mutant.

In VLHL PAI-1, the presence of two cysteines promotes the disulfide bridge 355-S-S-197 holding the proximal hinge of RCL to the B-sheet A and stabilizes the active form of PAI-1 with residue 197 pulled towards 353. The modeling of Krüger et al indicates that when the parallel strands of sheet A (yellow, Fig. 3a) part, residues 197 and 355 could be as much as $18 \AA$ away during transformation into latency, suggesting that, in the case of the VLHL mutant, it would require breaking of the -S-S- bridge. Hence, without the reducing agent, the majority of molecules stay active but not necessarily in the extended form (Fig. 3a and b, lane 1 on gel from left, active 1 and active 2). However, under reducing conditions (upon addition of DTT) the RCL can easily retract into the latent 


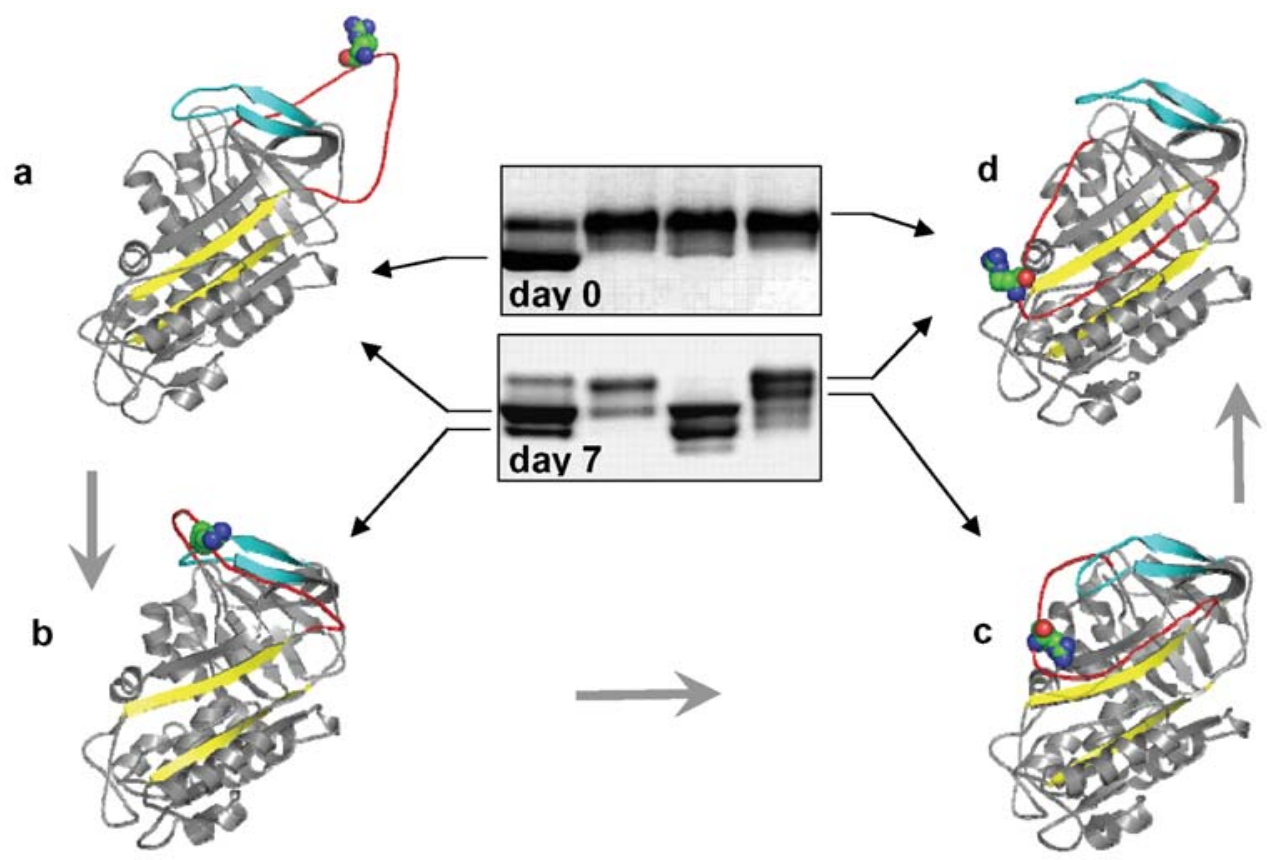

Figure 3. Ribbon models of (a) active 1; (b) active 2; (c) active 3 and (d) latent VLHL PAI-1. The RCL is shown in red, strands A3 and A5 in yellow and $\mathrm{s} 3 \mathrm{C} / \mathrm{s} 4 \mathrm{C}$ in light blue. The active site arginine is shown as spheres. Arrows point to the postulated structures of the different conformers of VLHL PAI-1.
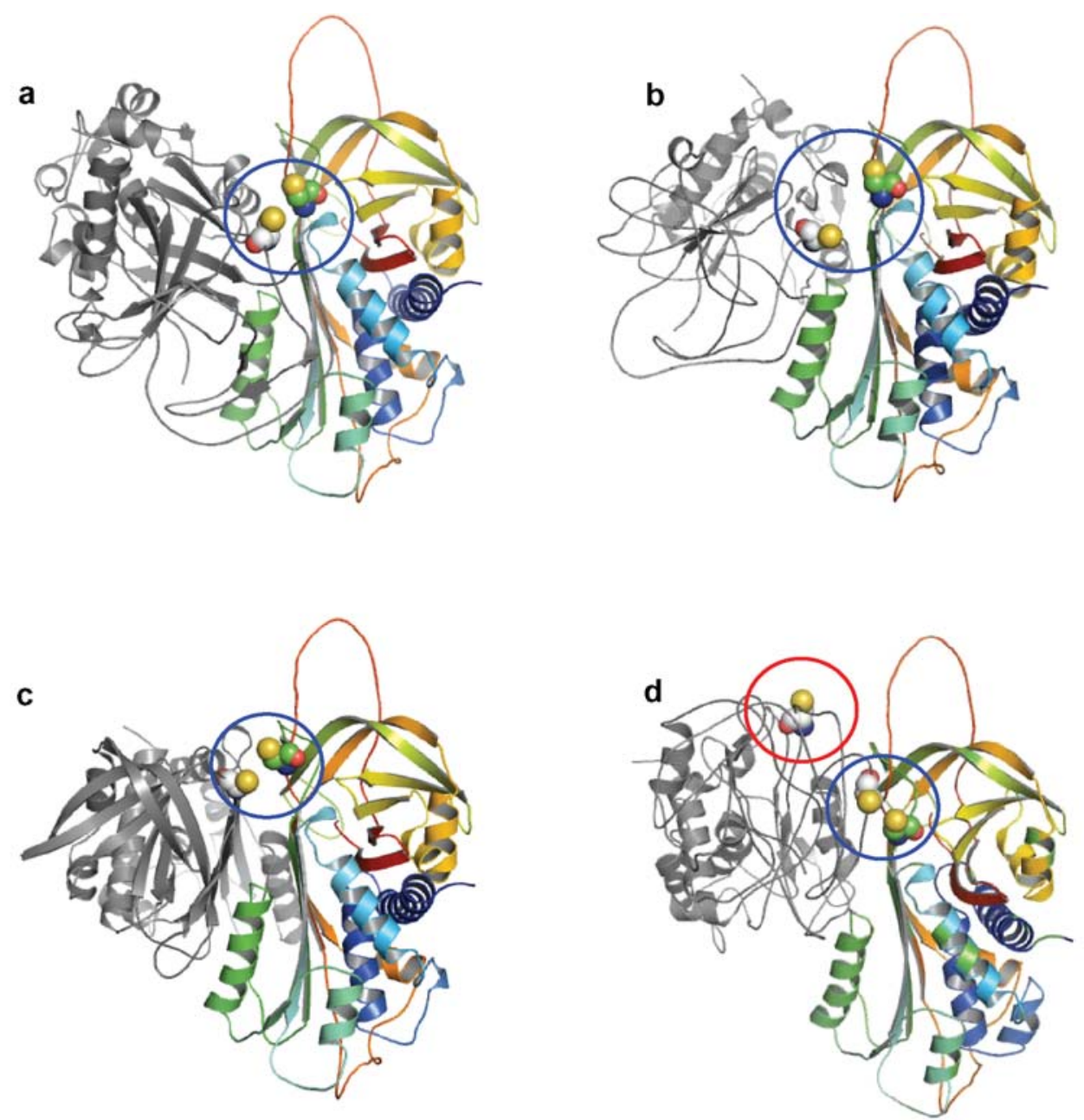

Figure 4. Dimers of VLHL PAI-1. The active form shown as a rainbow ribbon model acted as a receptor in the molecular modeling and the different conformers of VLHL PAI-1 shown as a ribbon model in gray acted as the ligand. All cysteines were reduced, and the only ones that could form a disulfide bridge are shown as spheres. Blue circles indicate dimer-forming cysteines and red trimer- or polymer-forming amino acids. (a) Active 1/active 2 dimer, (b) active 1/active 2 dimer model 2, (c) active 1/latent dimer, (d) active 1/active 3 dimer. 
position (Fig. 3d, lane 2 on gel), but with time and under aerobic conditions it can fully (Fig. 2, lane 3 on gel) or partially recover (Fig. 2, lane 4 on gel) when DTT is removed or degrades with time. PAI-1 RCL insertion into $B$-sheet $A$ of the serpin has been estimated to be a low energy event with $\Delta \mathrm{H}=-4.0 \mathrm{kcal} / \mathrm{mol}(24)$. In addition, it has been reported that the distribution of energy values for 706 known disulfide bonds is in range of $1.07-7.91 \mathrm{kcal} / \mathrm{mol}(25)$. The simulated transformation to latency includes bending of the 206-228 hairpin, swinging of the protein $\mathrm{C}$-terminus, shifting and various conformational changes in the nearby residues of the gate region. Although in the latent form cysteines 355 and 197 could be close enough in space to re-establish the -S-Sjunction when pushing 356-366 out of the sheet, the RCL may not be able to pass the gate region in the opposite direction to be fully extended. The hinge arginine could be engaged in strong salt bridges between Lys266...Arg $379 . .$. Glu401, thereby blocking the gate and forming a clamp between sheet $\mathrm{B}$ and the C-terminus. However, the RCL might adapt a conformation with a favorable steric condition to regain uPA/tPA activity (Fig. 3c, active 3 ).

Thus, the active form of VLHL PAI-1 could have more than one conformer: i) active 1 with the flexible RCL stretched out, traditionally considered as the active form and (26) active 2 or iii) active 3 with the RCL arrested in the proximity of the hairpin gate region, where Arg369 and Met370 of P1, P1' may still be accessible to react with tPA or uPA.

Heat treatment of VLHL PAI-1. Katagiri et al showed that latent wild-type PAI-1 can be reactivated by heat treatment up to $100^{\circ} \mathrm{C}$ for up to $2.5 \mathrm{~min}$ and regenerate about $70 \%$ of the initial activity (27). Heat treatment of sample 3 did not significantly change the SDS gel appearance or the VLHL PAI-1 activity. However, heat treated samples showed signs of dimerization and polymerization, which was abolished with DTT treatment.

Polymerization of VLHL PAI-1. For some time it was a general belief that PAI-1 did not form polymers, which was quite unique among active serpins (28). However, it has been shown lately that PAI-1 could spontaneously form polymers at low $\mathrm{pH}$ or polymerization could be induced by small organochemical ligands $(22,29)$. Heating experiments with VLHL PAI-1 were based on the work of Katagiri et al, who have shown that latent wtPAI-1 can be reactivated by heating up $100^{\circ} \mathrm{C}$ for a short time (27). VLHL PAI- 1 can be reactivated starting at $60^{\circ} \mathrm{C}$ as shown in Fig. 1. Although one can easily notice that heat induces polymerization of VLHL PAI-1, only traces of VLHL PAI-1 polymers can be seen in heat-treated freshly purified protein (Fig. 1, panel a), but significantly more can be detected in VLHL PAI-1 stored for 7 days (Fig. 1, panel c). Subsequent treatment of polymers with DTT causes depolymerization. This strongly suggests that polymerization of our mutated PAI-1 involved the formation of disulfide bridges. To evaluate this possibility, we have done a series of docking calculations on different VLHL PAI-1 conformers. We have found that some VLHL PAI-1 conformers can associate into dimers with the same molecule or with one of a different shape. Some of them can be in a configuration in which two or more cysteines of different molecules are in close proximity. Cysteines belonging to different molecules in the dimer were too far apart to form a bridge at room temperature [Fig. 4, ranging from more probable $(7 \AA)$ to less probable $(13 \AA)$ as measured between $\mathrm{C} \alpha$ atoms]. However, when temperature rises and consequently the mobility of the protein molecules rises as well, the probability of polymerization of VLHL PAI-1 increases. The majority of molecules would form intramolecular disulfide bridges, but some can form intermolecular -S-S- leading to dimers or higher oligomers of different conformers, as can be seen in PAGE gels (Figs. 1 and 3).

The very long half-life of active VLHL PAI-1 is essential for its therapeutic applications. Understanding the process of extending the half-life of VLHL PAI-1 is critical to move this protein into clinical practice. Based on molecular modeling of wtPAI-1 and a VLHL PAI-1 homologous model, we conclude that this serpin cannot convert into the latent form without breaking a disulfide bridge (Cys 197-355) that is otherwise arresting this molecule in an active conformation. Even when the bridge is reduced, the molecule can spontaneously reform the disulfide bridge and stabilize VLHL PAI-1 molecules into conformers that exhibit inhibitory activity toward uPA. The other beneficial property of VLHL PAI-1 is its inability to polymerize by $-\mathrm{S}-\mathrm{S}$ - bonds, which always must be considered when free cysteines could be present. Polymerization of VLHL PAI-1 can be induced only under very anomalous conditions, such as high temperature and/or the presence of a reducing agent.

\section{Acknowledgements}

We thank Dr R. Hart (President, American Diagnostica Inc., Stamford, CT, USA) for discussions, his support and the chemicals used in this study. This study was supported in part by grants from American Diagnostica Inc. and the Frank D. Stranahan Endowment Fund for Oncological Research.

\section{References}

1. Dano K, Romer J, Nielsen BS, et al: Cancer invasion and tissue remodeling - cooperation of protease systems and cell types. APMIS 107: 120-127, 1999

2. Jankun J, Aleem AM, Selman SH, et al: Highly stable plasminogen activator inhibitor type one (VLHL PAI-1) protects fibrin clots from tissue plasminogen activator-mediated fibrinolysis. Int J Mol Med 20: 683-687, 2007.

3. Duffy MJ: Proteases as prognostic markers in cancer. Clin Cancer Res 2: 613-618, 1996.

4. Pawlak K, Pawlak D and Mysliwiec M: Urokinase-type plasminogen activator and metalloproteinase- 2 are independently related to the carotid atherosclerosis in haemo-dialysis patients. Thromb Res 121: 543-548, 2008.

5. Berkenpas MB, Lawrence DA and Ginsburg D: Molecular evolution of plasminogen activator inhibitor-1 functional stability. EMBO J 14: 2969-2977, 1995.

6. Chorostowska-Wynimko J, Swiercz R, Skrzypczak-Jankun E, Wojtowicz A, Selman SH and Jankun J: A novel form of the plasminogen activator inhibitor created by cysteine mutations extends its half-life: relevance to cancer and angiogenesis. Mol Cancer Ther 2: 19-28, 2003.

7. Lawrence DA, Olson ST, Palaniappan S and Ginsburg D: Serpin reactive center loop mobility is required for inhibitor function but not for enzyme recognition. J Biol Chem 269: 27657-27662, 1994.

8. Follo M and Ginsburg D: Structure and expression of the human gene encoding plasminogen activator inhibitor, PAI-1. Gene 84: 447-453, 1989. 
9. Pannekoek H, Veerman H, Lambers H, et al: Endothelial plasminogen activator inhibitor (PAI): a new member of the Serpin gene family. EMBO J 5: 2539-2544, 1986.

10. Stoop AA, Eldering E, Dafforn TR, Read RJ and Pannekoek H: Different structural requirements for plasminogen activator inhibitor 1 (PAI-1) during latency transition and proteinase inhibition as evidenced by phage-displayed hypermutated PAI-1 libraries. J Mol Biol 305: 773-783, 2001.

11. Jankun J, Aleem AM, Specht Z, et al: PAI-1 induces cell detachment, downregulates nucleophosmin (B23) and fortilin (TCTP) in LnCAP prostate cancer cells. Int J Mol Med 20: 11-20, 2007.

12. PyMOL v. 0.98. De Lano WL: The PyMOL molecular graphics system, 2005.

13. Baker NA, Sept D, Joseph S, Holst MJ and McCammon JA: Electrostatics of nanosystems: application to microtubules and the ribosome. Proc Natl Acad Sci USA 18: 10037-10041, 2001.

14. Sharp AM, Stein PE, Pannu NS, et al: The active conformation of plasminogen activator inhibitor 1, a target for drugs to control fibrinolysis and cell adhesion. Struct Fold Des 7: 111-118, 1999.

15. Tucker HM, Mottonen J, Goldsmith EJ and Gerard RD Engineering of plasminogen activator inhibitor-1 to reduce the rate of latency transition. Nat Struct Biol 2: 442-445, 1995.

16. Krüger P, Verheyden S, Declerck PJ and Engelborghs Y: Extending the capabilities of targeted molecular dynamics: simulation of a large conformational transition in plasminogen activator inhibitor 1. Protein Sci 10: 798-808, 2001.

17. Mustard D and Ritchie DW: Docking essential dynamics eigenstructures. Proteins 60: 269-274, 2005.

18. Ritchie DW and Kemp GJ: Protein docking using spherical polar Fourier correlations. Proteins 39: 178-194, 2000.

19. Basrur V, Yang F, Kushimoto T, et al: Proteomic analysis of early melanosomes: identification of novel melanosomal proteins J Proteome Res 2: 69-79, 2003.
20. Tozser $\mathbf{J}$ and Berta A: Plasminogen activator inhibitors in human tears. Acta Ophthalmol (Copenh) 69: 426-431, 1991.

21. Lee HJ and Im H: Purification of recombinant plasminogen activator inhibitor- 1 in the active conformation by refolding from inclusion bodies. Protein Expr Purif 31: 99-107, 2003.

22. Jankun J, Specht Z, Szkudlarek M, et al: Plasminogen activator inhibitor-1 is locked in active conformation and polymerizes upon binding ligands neutralizing its activity. Int J Mol Med 17: 437-447, 2006

23. Li S-H, Gorlatova NV, Lawrence DA and Schwartz BS: Structural differences between active forms of plasminogen activator inhibitor type 1 revealed by conformationally-sensitive ligands. J Biol Chem 283: 18147-18157, 2008.

24. Boudier C, Gils A, Declerck PJ and Bieth JG: The conversion of active to latent plasminogen activator inhibitor- 1 is an energetically silent event. Biophys J 88: 2848-2854, 2005.

25. Dombkowski AA: Disulfide by design: a computational method for the rational design of disulfide bonds in proteins. Bioinformatics 19: 1852-1853, 2003.

26. Iishi $H$, Tatsuta $M$, Baba $M$, Yano $H$, Uehara $H$ and Nakaizumi $A$ : Suppression by amiloride of bombesin-enhanced peritoneal metastasis of intestinal adenocarcinomas induced by azoxymethane. Int J Cancer 63: 716-719, 1995.

27. Katagiri K, Okada K, Hattori H and Yano M: Bovine endothelial cell plasminogen activator inhibitor. Purification and heat activation. Eur J Biochem 176: 81-87, 1988.

28. Zhou A, Faint R, Charlton P, Dafforn TR, Carrell RW and Lomas DA: Polymerization of plasminogen activator inhibitor-1. J Biol Chem 276: 9115-9122, 2001.

29. Pedersen KE, Einholm AP, Christensen A, et al: Plasminogen activator inhibitor-1 polymers, induced by inactivating amphipathic organochemical ligands. Biochem J 372: 747-755, 2003. 